\title{
Asymmetric priming effects in visual processing of occlusion patterns
}

\author{
GIJS PLOMP and CEES VAN LEEUWEN \\ Brain Science Institute, RIKEN BSI, Wako-Shi, Japan \\ and University of Sunderland, Sunderland, England
}

\begin{abstract}
In three experiments, we examined the effect of temporal context in amodal completion of partly occluded nontarget figures. In a primed same-different task, test pairs were preceded by a sequence of two primes, one of which was a single, the other a composite figure. Single figures reappeared in the composite ones, which also contained a square that could be viewed, alternatively, as an occluder or as yielding a mosaic fit to the other shape. To measure context influences between single and composite figures, both of which were nontargets, we studied their combined effect as primes on the test pairs of the same-different task, expecting that congruency between both primes should lead to a superadditive priming effect on the task. We found that single figures presented first provided facilitatory context for local and global occlusion as well as for mosaic interpretations of subsequently presented composite figures. These effects occurred only when the composite figure was presented briefly (50 msec). No superadditive facilitation occurred when composite figures were presented first and single figures followed them. The restriction of the effect to short presentations and its temporal asymmetry were taken as evidence that prior context biases possible occlusion interpretations during the process of completion, rather than afterward.
\end{abstract}

\section{Completion and Context}

When part of an object is hidden behind an occluder, a mental operation takes place that makes the object appear as whole and complete. This ubiquitous phenomenon is called amodal completion, because it is unaccompanied by a visual sensation of the missing part (Michotte, Thinès, \& Crabbé, 1964). The perceiver has, however, a clear and distinct idea of what figure lies behind the occluder. Amodal completion has been studied extensively using the methods of experimental phenomenology (Kanizsa, 1979). In a typical experiment, a geometrical shape is partly occluded and two or more unoccluded alternatives are presented, from which one has to be chosen. In this manner, reliable preferences for completions are observed (Buffart \& Leeuwenberg, 1981; Buffart, Leeuwenberg, \& Restle, 1983). A restriction of these studies, however, is that such methods fail to indicate the mechanisms that are responsible for the completion process. Reaction time (RT) studies have proven more suitable for this purpose (Gerbino \& Salmaso, 1987; Sekuler \& Palmer, 1992). We therefore investigated the underlying mechanisms of amodal completion in a primed same-different task.

Starting from phenomenal descriptions of completed figures, several factors can be identified that might, in

The authors are grateful for the helpful comments and suggestions of two anonymous reviewers, which led to substantial improvement of a previous version of the manuscript. The authors are affiliated with the Laboratory of Perceptual Dynamics at the RIKEN Brain Science Institute and with the Department of Psychology at Sunderland. Correspondence relating to this article may be sent to C. van Leeuwen, 2-1 Hirosawa, Wako-Shi, Saitama 351-0198, Japan (e-mail: ceesvl@brain.riken.jp). principle, account for how completed representations of incomplete objects are made. Two of these factors relate to the structure of the visible parts. The contours of the parts can sometimes be simply interpolated to achieve the completion (Kanizsa \& Gerbino, 1982; Kellman \& Shipley, 1991; Shipley \& Kellman, 2003). This means that features local to where the shape disappears behind the occluder determine how it is completed. There are also situations in which completion is based on global properties of the partly occluded figure (Sekuler, Palmer, \& Flynn, 1994; van Lier, Leeuwenberg, \& van der Helm, 1995). Global completion may involve maximizing the symmetry of the completed shape.

Local and global completion mechanisms may be hard to separate physiologically. Local contour interpolation processes start early in visual processing and can be achieved fast and independently of task-specific attention. These early processes, however, are sensitive to nonlocal properties of the visual structure as well (Altmann, Bülthoff, \& Kourtzi, 2003; Kamitani \& Shimojo, 2004; Nikolaev \& van Leeuwen, 2004), possibly through lateral interactions within cortical areas V1 and V2. Perhaps because of this similarity, no difference in processing speed may be detectable between local and global completions, even with sensitive measures based on eyetracking (Plomp, Nakatani, Bonnardel, \& van Leeuwen, 2004).

In addition to local and global completion interpretations, an occluded figure can alternatively be interpreted as a two-dimensional figure. In that case, the interpretation resembles a mosaic, a cutout figure that adjoins another. Every partly occluded figure has, at least in principle, such a mosaic interpretation. We will, therefore, adopt the neu- 
tral term composite figures for patterns that have occlusion as well as mosaic interpretations. Several researchers have utilized the possibility of giving a mosaic interpretation to composite figures. When participants search for cutout circles in a display containing whole circles and target composite figures, prolonged presentation of the display typically preempts detecting the mosaic shapes contained in the composite figures (Rensink \& Enns, 1998). This suggests that the mosaic shapes become quickly and automatically represented as occluded figures. The search for mosaics is easy, however, when search displays are shown briefly and masked immediately (Rauschenberger $\&$ Yantis, 2001). These findings suggest that completion moves through distinct stages and that a "mosaic stage" may precede completion (Sekuler \& Palmer, 1992).

Most research has focused on completion in relation to stimulus properties and has considered these properties to be context independent. Some have argued, in addition, that completion proceeds through separate stages. This happens automatically, in a context-independent manner. The context independency of occlusion processes has recently come under fire, however. The notion that completion goes through these distinct stages has been contrasted with the idea that alternative interpretations, mosaic as well as occlusion, are generated in parallel (Bruno, Bertamini, \& Domini, 1997). In order to explain the preemption effect, this view proposes that different interpretations may predominate because of the effects of context (Peterson \& Hochberg, 1983). Nontargets in a search display have been shown to influence the interpretation of the targeted composite figure (Rauschenberger, Peterson, Mosca, \& Bruno, 2004). In Rauschenberger et al.'s study, search for a whole circle was impeded when the surrounding figures were notched circles. These results directly demonstrated the role of spatial context on the completion process and are in accordance with parallel processing of mosaic and occlusion interpretations rather than a separate mosaic stage.

The effect of spatial context is present for long presentation times of the display only (Rauschenberger et al., 2004). This may indicate that context influences occur late in the process, but it may also reflect the time needed to process the surrounding figures in the display. Thus, we cannot conclude from this study whether context influences occur early, during the process of completion, or afterward, in a postperceptual decision process. With spatial context, this problem cannot be avoided, since spatial context by definition involves the concurrent processing of other figures. For this reason, the temporal domain may be more suitable for observing whether context effects influence the ongoing completion process or have their influence afterward.

Effects of temporal context on completion have recently been reported. The direction of ambiguous motion behind an occluder can be biased by the figure seen prior to it (Joseph \& Nakayama, 1999). In addition to the possible role of retinal afterimages in this paradigm, there may be differences in the way ambiguity is resolved in the completion of occluded motion versus completion of static figures. The present study is focused on the role of preceding information in static occluded figures.

For static composite figures, prior exposure can induce completion interpretations that are otherwise unlikely (Beller, 1971; Sekuler \& Palmer, 1992; Zemel, Behrmann, \& Mozer, 2002). In Zemel et al.'s study, participants saw two composite figures in a display and judged whether some local features of them (i.e., a number of "bumps") were the same or different. The composite figures at first did not suggest an occlusion interpretation, but after exposure to such an interpretation this changed. Prior exposure facilitated the same-different task because the composite figure was grouped as a complete figure lying behind another, instead of as two disjoint figures abutting a rectangle. This result indicates that temporal context influences amodal completion, but it does not tell us how this influence is achieved - that is, whether context influences the completion process itself or acts to reinterpret the figure after it has been perceived. Such results, therefore, do not allow us to determine whether perceptual completion processes or later decision stages are influenced by context.

The reason that we cannot distinguish between perceptual and postperceptual effects in the Zemel et al. (2002) study is that in this and related studies, the targets of prior exposure coincide with the test figures. Instead of priming in completion, we may therefore be observing priming of some later stage in the perceptual process as well as of other processes involved in the task. To study the effect of temporal context, it is preferable to have the target of priming separated from the figures on which the task is performed. Only in that way can we be reasonably sure that the influence is on visual processes and not on decision- or response-related ones.

Separation of test and target figures can be achieved by extending the primed matching paradigm. In this paradigm, prior exposure decreases RT in a same-different task (Beller, 1971). Typically, the presentation of a composite figure facilitates responses to test pairs containing a possible interpretation of the composite (Sekuler \& Palmer, 1992). For example, in Sekuler and Palmer's study the presentation of a partly occluded circle facilitated responses to a subsequent test pair containing two circles. This fact suggests that the partly occluded figure was completed and primed the visual system for the occurrence of circles in the test pair. However, a problem still remains in that study, in that the test figures were also the target of the priming. We therefore extended this paradigm in the following way. Before the test pair, we presented a sequence of two figures. In principle, therefore, both could independently prime the test figures and thus facilitate the task. Of crucial interest, however, was the interaction between the first and second figures. Would the first one influence the visual processing of the second, even though neither of them were targets of the task? If such an effect was present, it would result in a superadditive facilitation by the first and second figures on the task (Stins \& van Leeuwen, 1993), and this result could be understood as an indicator that the first nontarget figure primed the second. 
In particular, imagine that the first of the preceding figures was a single, unoccluded figure and the second a composite. If the single figure primed the composite one, we would observe a superadditive effect when both were congruent to the test pair. Because the target of priming, the composite, was not part of the task, the superadditivity could be attributed to differential processing of the composite figure and not to facilitation of processes involved in the same-different decision. We must note, however, that the presence of such an interaction effect does not specify the direction of the influence. In Experiment 1, we examined whether a simple figure can influence the processing of a composite one, and in Experiments 2 and 3, we addressed whether the result was specific to the order of the single and composite figures.

If several interpretations of a composite figure are generated more or less in parallel and context serves to prime these, we may ask when this selection takes place. A potential determinant may be whether completion can finish on the basis of the immediate visual information. Given the quick and mandatory character of amodal completion (Rensink \& Enns, 1998), context effects may be relatively weak relative to the directly available visual information, and they may therefore be more prominent when a composite is presented only briefly, when completion has no time to finish. To establish under what conditions context is most effective, we varied the presentation time of the composite figures. In one condition, the composite figure was presented for $50 \mathrm{msec}$, so that a complete representation was unlikely to be formed while the figure was on screen. In another, the figure was presented for $500 \mathrm{msec}$. In that case, amodal completion processes most likely would end while the figure was still on the screen. Our manipulation should help localize the influence of context in amodal completion.

\section{EXPERIMENT 1}

The experiment was based on a primed matching paradigm, with two figures successively preceding a samedifferent task. The first was a single and the second a composite figure. Single figures were possible interpretations of a composite figure, but not always of the following one. Both figures could be congruent or incongruent to the test pair. When a figure is congruent to the test pair, RTs are expected to decrease (Beller, 1971). These congruency effects were evaluated on RTs to same test pairs. Congruency between the single figure and the test pair meant that the former was identical to a figure in the latter, except for the angle of rotation. We rotated the test pair relative to the previous displays in order to ensure that the figures' lowlevel stimulus attributes were not constant between the displays. Between the composite figure and the test pair, congruency meant that a figure in the test pair had the same shape as one of the interpretations of the composite (for instance, if the composite was a partly occluded circle, the test pair would be congruent if it contained a circle). We independently varied the two congruencies in a factorial design. The critical condition was when both preceding figures were congruent to the following test pair - that is, when the single figure was a possible interpretation of the following composite. If this biased the interpretation of the composite, then the combined effect of the two figures on RT would be larger than their summed individual effects. This contextual influence is expected to be strongest when the composite is presented too briefly for completion processes to finish, given the available visible information.

\section{Method}

Participants. There were 17 participants in the experiment, who all received a small payment in return for their efforts. Ten of them were male and 7 female, and all had normal or corrected-to-normal vision. Their age range was $20-29$ years, with a mean of 23 years. Each participant provided written informed consent to the procedures as required by the RIKEN ethics committee.

Materials and Design. Figure 1 shows the stimuli. They consisted of four composite figures, with three single figures for each of them, corresponding to the possible alternative interpretations of that composite figure. One possible occlusion interpretation was always a local completion (based on the continuation of lines at the point of occlusion) and the other a global one (based on maximizing the number of symmetry axes of the partly occluded figure). The average amount of occlusion was $30 \%$ across all occlusion interpretations; for half of the composites, the global completion had

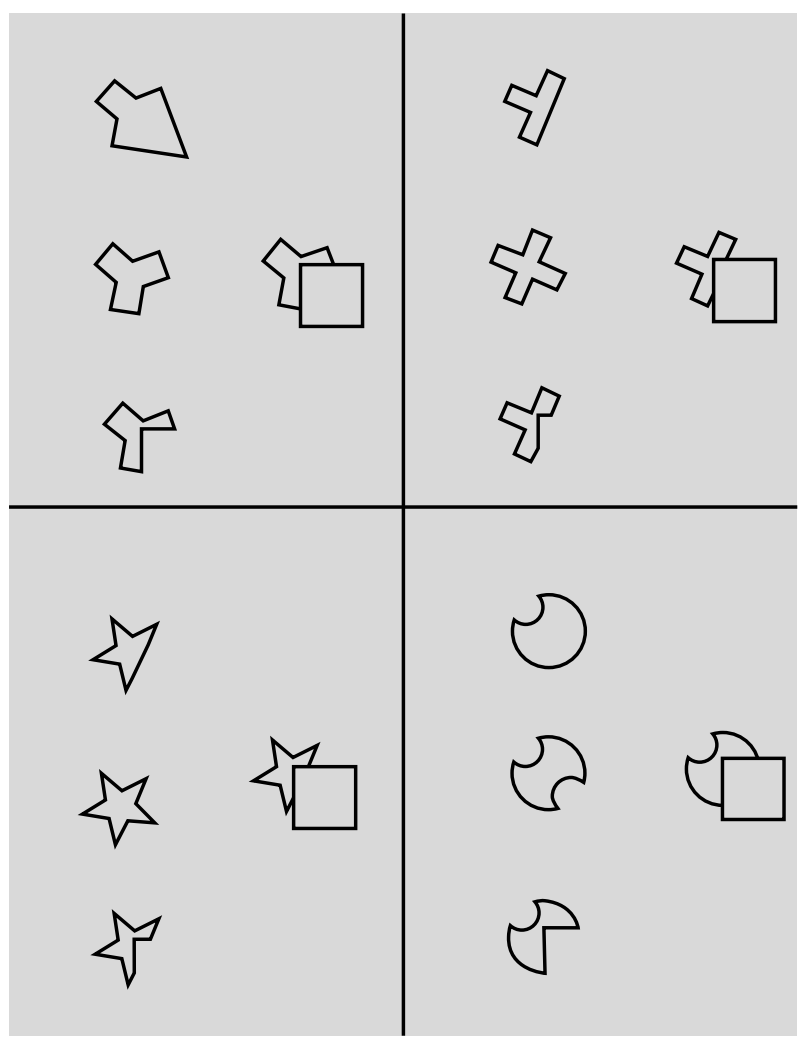

Figure 1. The four ambiguously occluded figures (right side of each panel) and their respective local, global, and mosaic interpretations (the figures in the top left panel are based on figures from van Lier, Leeuwenberg, \& van der Helm, 1995). 


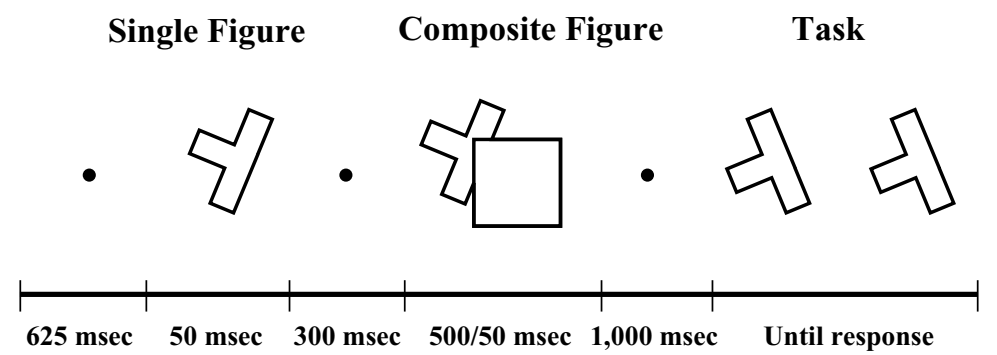

Figure 2. Example of a trial in which both the single and composite figures are congruent with the following same task.

the largest occluded surface, and for the other half the local one did. The third possible interpretation of each composite was as a mosaic (a 2-D cutout figure). The single figures corresponding to a global completion were called, by association, global figures; those corresponding to a local completion, local figures; and those corresponding to a mosaic interpretation, mosaic figures. Stimuli for the same-different task always consisted of a pair of same or different global, local, or mosaic figures.

A trial began with a fixation dot. On each trial, three displays were sequentially presented, showing stimuli on a uniformly gray background. In the interstimulus interval (ISI) between the displays, the fixation dot was shown on the screen. The first display showed a single figure, the second a composite figure, and the last a test pair. The single figure in the first display was centered on the screen and was presented for $50 \mathrm{msec}$. An ISI of $300 \mathrm{msec}$ separated the first and second displays. The composite figure in the second display was placed such that the contours it shared with the single figure in the first display appeared in the same locations. Composite figures were presented for either short $(50-\mathrm{msec})$ or long $(500-\mathrm{msec})$ durations. In the short presentation condition, the composite figure disappeared while the completion process was still ongoing (Sekuler \& Palmer, 1992). In the long condition, enough time was available for completion to finish while the figure was on screen. Between the second and third displays was an ISI of $1 \mathrm{sec}$. The third display contained the test pair of two single figures, which stayed on screen until the participant indicated whether they were the same or different. Figure 2 provides an overview of a trial.

All stimuli could occur in four orientations that differed by $90^{\circ}$ from each other. The two figures in a test pair appeared in identical orientations to allow a decision based on low-level stimulus criteria, enabling a rapid response. The single and composite figures always had identical orientations within a trial. This, too, was done in order to allow for low-level interactions between the first and the second stimulus, since, as argued, low-level processes may play an important role in local as well as global completions. The figures in the test pair were always rotated $90^{\circ}$ relative to the preceding figures. The difference in orientation from the preceding stimuli was introduced in order to minimize the influence of low-level processes on priming of the test figures. In addition, in order to enable all completion processes for the composite figure to finish before the onset of the task, the ISI between the composite figure and the test pair was made longer than traditionally has been the case. Whereas the ISI has previously been as short as $10 \mathrm{msec}$ (Sekuler \& Palmer, 1992), we extended it to $1 \mathrm{sec}$, enabling more than sufficient time for completion to finish. In addition, this interval minimized the influence of retinal aftereffects on the task. Masking can eliminate the effects of retinal afterimages as well, but we refrained from using it in our experiment because it may also disrupt the completion process.

The critical factor in the experiment was the congruency of the single and composite figures to the test pair. The single figure was congruent to the test pair when one of the test figures was the same as the single figure; the composite figure was congruent to the test pair when a single figure corresponding to one of its interpretations appeared at test. The congruency of the figures was independently varied, resulting in four congruency conditions. In one of them, both the single and composite figures were congruent to the test pair; in two others, only one of them was congruent; and in the fourth condition, both were incongruent to the test pair. In the latter case, the single figure was related to a different composite figure than were the ones in the test pair. Across the four conditions, at least one of the preceding figures was congruent to the task stimuli in three out of four trials. Thus, the preceding figures were predictive of one of the test shapes. The relevance of the preceding figures encouraged the participants to pay attention to them, even though those figures were not the targets of the experimental task. Examples of a trial for each congruency condition are given in Figure 3.

In every congruency condition, each composite figure was preceded once apiece by a local, a global, and a mosaic figure. Thus, there were $12(3 \times 4)$ combinations of single and composite figures within a congruency condition. Across the three conditions in which
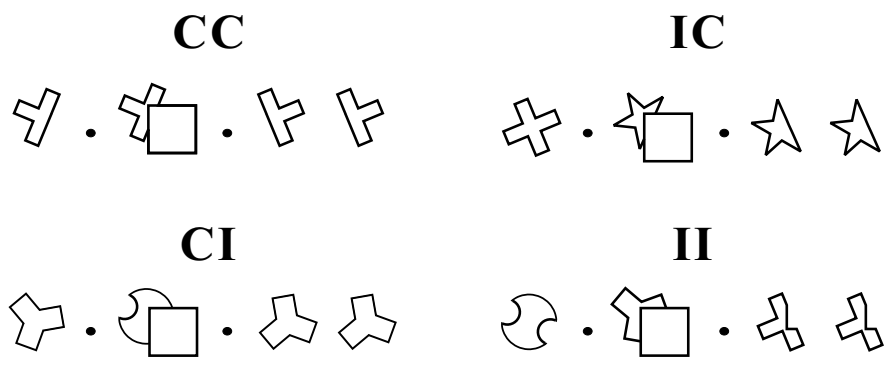

Figure 3. Examples of the four congruency conditions. On each trial, single and composite figures were either congruent (C) or incongruent (I) to the test pair. 
at least one figure was incongruent to the test pair, each composite figure co-occurred with each possible single figure of the other composite figures.

Each single-composite sequence in a condition could be followed by a same or a different test pair. The same pairs consisted of two identical single figures, either local, global, or mosaic. Per unique sequence, each same pair was matched to a corresponding different pair, such that when a same pair occurred with a given sequence, the different pair also consistently appeared with it. This ensured that the occurrence of a different pair with a given sequence was balanced in the same manner as the occurrence of same pairs. The different pairs were created by substituting one figure of the same pair with a single figure of the same type (local, global, or mosaic) that was congruent with a different composite figure. The occurrence of single figures in different pairs was balanced across the congruency conditions so that all single figures occurred equally frequently.

To summarize, the 4 congruency conditions and their 12 combinations of single and composite figures constituted the 48 unique sequences of the experiment. The composite figure could be presented for 50 or $500 \mathrm{msec}$, and the sequence could be followed by a same or a different test pair. The total design thus consisted of $192(48 \times 2 \times 2)$ unique trials, which were presented 10 times to each participant.

Apparatus and Procedure. A personal computer displayed the stimuli on a $160-\mathrm{Hz}, 17-\mathrm{in}$. CRT monitor, the center of which was leveled to the height of the participant's eyes. The exact timing of the presentation was controlled with a page-cycling routine by a Visual Stimulus Generator (Cambridge Research Systems). The participants sat in a supportive chair $90 \mathrm{~cm}$ from the screen. The largest stimuli, the composite figures, occupied approximately $2.5^{\circ}$ of visual angle; the centers of the two figures in the test pair were separated by about $4.5^{\circ}$. The display luminance was $35 \mathrm{~cd} / \mathrm{m}^{2}$ on average. A participant's response consisted of pressing buttons on a response pad. Half of the participants gave same responses with their left hand, the other half with their right hand, and after their response they received feedback in the form of a smiling or frowning icon. All trials were self-paced and were initiated by a buttonpress.
The experiment consisted of four sessions that were split over 2 days. A session lasted about $40 \mathrm{~min}$, and a refreshment break was scheduled between sessions on each day. On the first day, an additional small practice session acquainted the participants with the procedures. All trials were presented in a random order that was determined before each session and was different for each participant.

\section{Results}

The error rate in the experiment was $4.1 \%$ overall. The analysis concerned responses to same trials only. None of the individual data showed signs of a speed-accuracy trade-off, and error rates therefore were not analyzed further. We took the RTs of the correct responses in the range between 250 and 3,000 msec. This range was chosen because their reciprocals were normally distributed. The analysis was performed on these reciprocals, ensuring a robust analysis (Hoaglin, Mosteller, \& Tukey, 2000). The participants differed in their overall response speed and their mean responses to the four stimulus classes. ${ }^{1}$ These differences were modeled as random effects in a mixedeffects model (Pinheiro \& Bates, 2000) as implemented in $\mathrm{R}$ (www.R-project.org). In this type of analysis, the random effects (e.g., interparticipant variance) are estimated from the data and taken into account when modeling the effects of interest, the fixed effects. The fixed effects were single figure (congruent/incongruent to the test pair), composite figure (congruent/incongruent to the test pair) and test type (local, global, and mosaic) and were evaluated in an ANOVA.

In the 50-msec condition, there were main effects of single figure $[F(1,7600)=51.01, p<.001]$ and composite figure $[F(1,7600)=12.56, p<.001]$. In both cases, con-

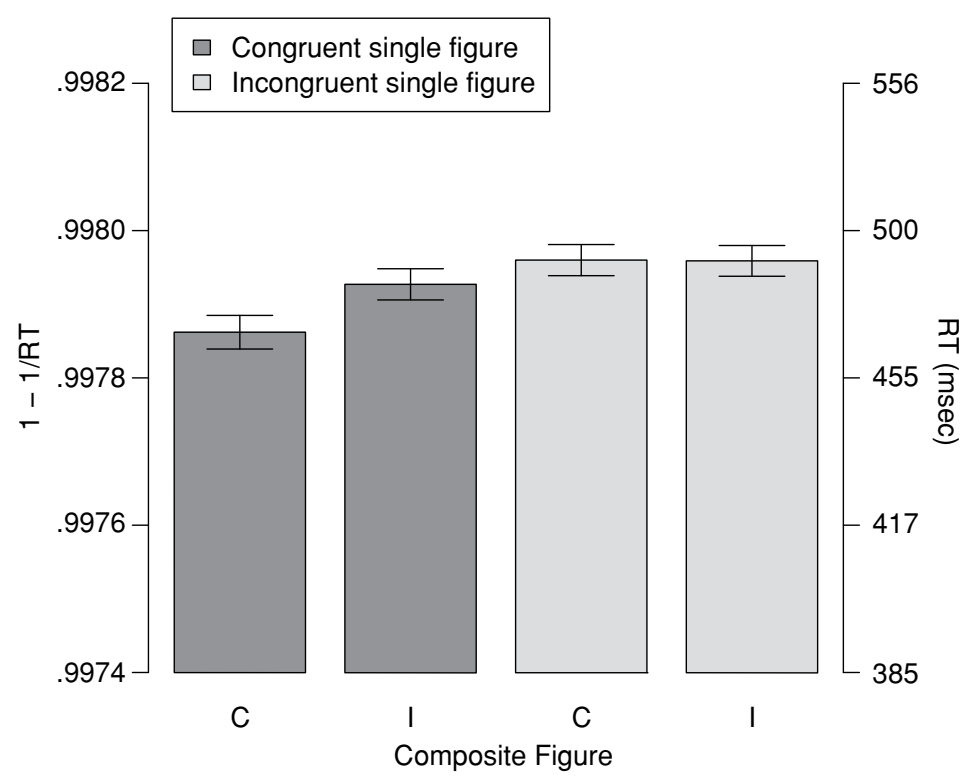

Figure 4. Mean and $95 \%$ confidence intervals of reaction times (RTs) for congruent and incongruent single and composite figures in Experiment 1. The $x$-axis denotes congruency of the composite figure (congruent $[\mathrm{C}]$ or incongruent $[\mathrm{I}])$. 
Table 1A

Mean Reaction Times (RTs, in Milliseconds; With Standard Deviations) to Global (G), Local (L), and Mosaic (M)

Test Pairs in Experiment 1 for Congruent (C) and Incongruent (I) Single and Composite Figures, for the Short (50-msec) Presentation Condition

\begin{tabular}{ccccc}
\hline & & & \multicolumn{2}{c}{ RT } \\
\cline { 4 - 5 } Single & Composite & Test Type & $M$ & $S D$ \\
\hline C & C & G & 471 & 130 \\
& & L & 487 & 125 \\
& I & M & 540 & 159 \\
& & G & 485 & 119 \\
& & L & 504 & 137 \\
I & M & 551 & 189 \\
& C & G & 495 & 126 \\
& & L & 507 & 129 \\
& & M & 561 & 163 \\
& I & G & 500 & 139 \\
& & L & 507 & 150 \\
& & M & 559 & 173 \\
\hline
\end{tabular}

gruency increased the reaction speed. The main effects of single and composite figure also interacted $[F(2,7600)=$ $10.62, p=.001]$; the congruency of the composite figure shortened RTs only when the single figure was congruent as well (see Figure 4). Test type influenced response speed, as well $[F(2,7600)=225.64, p<.001]$; global test pairs evoked the fastest responses, then local ones, and mosaic pairs gave rise to the slowest responses. Mean RTs for the short $(50-\mathrm{msec})$ presentation times are listed in Table 1A.

In the 500-msec condition, the congruency of the single and composite figures affected response speed $[F(1,7622)=29.26, p<.001$, and $F(1,7622)=8.66, p<$
.01 , respectively], but there was no interaction between the two. The main effect of test type was qualitatively similar to that in the $50-\mathrm{msec}$ condition $[F(2,7622)=$ $224.86, p<.001]$, but it interacted with single-figure congruency $[F(2,7622)=4.98, p<.01]$. Responses to local and global test pairs benefited from a congruent preceding single figure, whereas mosaic pairs (as depicted in Figure 5) did not, on average. No other effects reached significance. Mean RTs for long (500-msec) presentations are listed in Table 1B.

\section{Discussion}

The main effect of test type on RT (i.e., that mosaic pairs elicited longer RTs than did the other pairs) can be understood by the complexity of their shapes. This effect, as well as the main effects of the congruency of single and composite figures, is intuitive and similar for both presentation durations. The pivotal effect, however, was the interaction between the two congruency effects in the short presentation condition. Although the composite figure was still clearly visible, it did not prime the test figures on its own. However, when single and composite figures were both congruent to the test pair, the decrease in RTs was larger than the sum of their individual effects; for short presentations, the composite figure facilitated responses when the preceding single figure was one of its interpretations. We may worry about the absence of a main effect of congruency of the composite figure. But note that it was presented only briefly and was interposed between two other stimuli. This suggests that the composite figure can contribute a priming influence only when its interpretation is strengthened by the preceding figure.

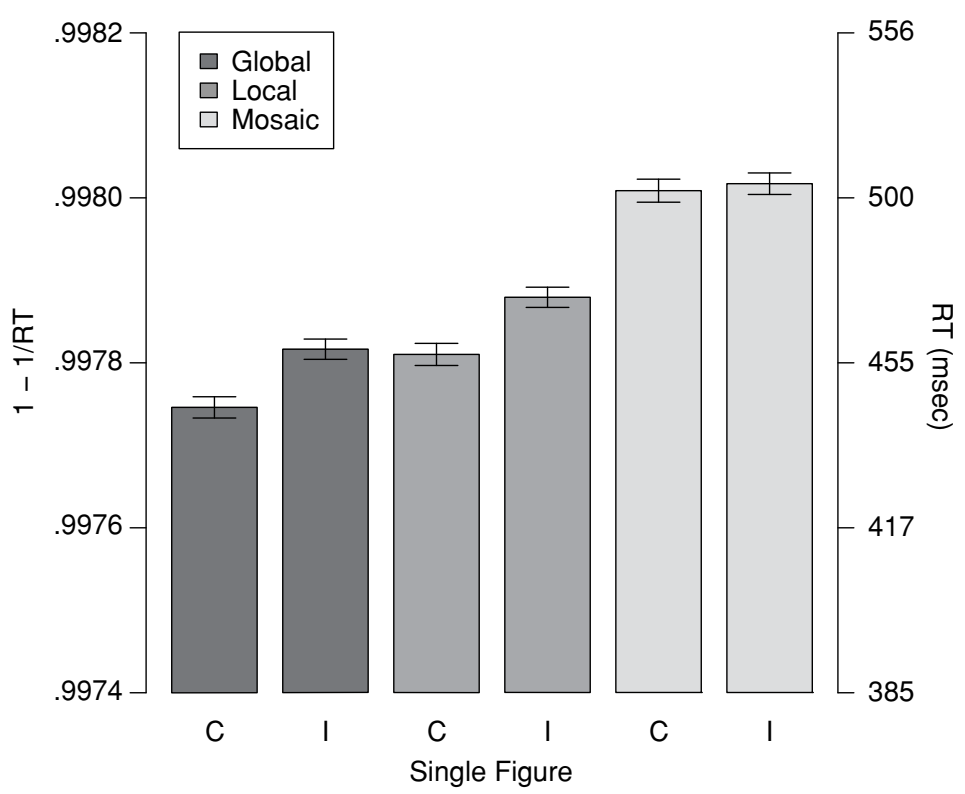

Figure 5. Mean and 95\% confidence intervals of reaction times (RTs) for the interaction between test type (local, global, or mosaic) and congruency (congruent $[\mathrm{C}]$ or incongruent $[\mathrm{I}])$ of the single figure in the long $(500-\mathrm{msec})$ presentation condition of Experiment 1. 
Table 1B

Mean Reaction Times (RTs, in Milliseconds; With Standard Deviations) to Global (G), Local (L), and Mosaic (M) Test Pairs in Experiment 1 for Congruent (C) and Incongruent (I) Single and Composite Figures, for the Long (500-msec) Presentation Condition

\begin{tabular}{ccccc}
\hline & & & \multicolumn{2}{c}{ RT } \\
\cline { 3 - 5 } Single & Composite & Test Type & $M$ & $S D$ \\
\hline C & C & G & 463 & 112 \\
& & L & 480 & 127 \\
& I & M & 532 & 144 \\
& & G & 468 & 111 \\
& & L & 486 & 127 \\
I & C & G & 545 & 164 \\
& & L & 476 & 122 \\
& & I & 495 & 132 \\
& & G & 539 & 151 \\
& & L & 488 & 128 \\
& & M & 497 & 118 \\
& & & 538 & 160 \\
\hline
\end{tabular}

This strengthening happens when the first and second stimuli are both congruent to the test figures, and therefore congruent to each other; the strengthening that the composite underwent involved biasing it toward one of its interpretations. In other words, its ambiguity needed to be resolved by a prior biasing stimulus in order for it to have a priming influence on the next stimulus. It is remarkable that this effect persisted to the presentation of the test figures, despite the long interval between the preceding stimuli and the task and the difference in orientation between prime and task figures. Because of these, the result cannot be attributed to low-level processes involving retinal image or visual persistence after the presentation of the composite.

We might, however, consider whether it is a product of cognitive deliberation - for instance, a result of participants' efforts to infer the relationship between the first and second stimuli. The interval between the stimuli and the task $(1,000 \mathrm{msec})$ is long enough, in principle, to do so, and therefore the current paradigm cannot rigorously exclude this possibility. Note, however, that the interaction was restricted to short presentations of the composite figure. This speaks against explanations in terms of cognitive deliberation, which is equally likely for long and for short presentations of the composite. For similar reasons, it is unlikely that the effect is based on response biases, which would not be undone by longer presentations of the composite figure. We may, therefore, interpret the superadditive interaction as an influence of context during the process of amodal completion. The absence of the interaction for the longer presentation duration of the composite indicates that continued incoming visual information can override this influence. The results, therefore, provide evidence that the influence of priming occurs during the completion process, rather than in cognitive processes afterward.

The result obtained is similar to the preemption effect in visual search (Rauschenberger et al., 2004), insofar as the directly available information can override initial bi- ases in the ongoing processing of the composite figure for longer presentations. The result differs from preemption in visual search, however, because in that process the visually available information in the surrounding context biases the interpretation, whereas in the present experiments the visually available information in the composite figure did not favor one particular interpretation over another. As a consequence, we observed context effects for short rather than for long presentations of the composite figure, effects that involved priming of all three possible alternative interpretations - the local and global occlusions as well as the mosaic.

For long presentation times, congruency facilitated responses to local and global but not to mosaic test pairs. This may suggest that after completion has finished, the representation of the preceding mosaic figure is deleted from memory. Thus conceived, the result is consistent with the preemption effect for long presentations in visual search (Rauschenberger \& Yantis, 2001). We will test this hypothesis in the next experiment, with the temporal order of the single and composite figures reversed. In this reversed order, the single figure will come right before the task, so we may expect to see priming effects of global, local, and mosaic figures, and therefore a main effect of single-figure congruency.

The reversal of first and second stimuli will allow us to control for an important alternative explanation. In this explanation, the interaction for short presentations indicates that priming does occur during completion, but such priming is irrelevant to the completion process itself. In Experiment 1, first and second figures were presented in the same position to enable interactions between low-level processes possibly involved in the completion process. However, it is possible that the priming of low-level features had nothing to do with the completion process, and if so, the interaction should be preserved when the order between the first two figures is reversed.

The alternative hypothesis could also explain the absence of priming for the mosaic figure under long presentation conditions. This figure is the only one that has an overlapping feature with any composite figure: Its straight edge is present in all subsequent composite figures and occurs in the same position on the screen. The long exposure of the second stimulus may have grouped this feature with the square in the composite figure, a kind of "feature hijacking." If such feature hijacking exists, it is not clear, however, how it would behave under reversal of the first and second stimuli.

Reversing the order of the single and composite figures will enable us to test whether the interaction between first and second stimuli under short presentation times is direction specific. The hypothesis assumes that a single figure primes the composite, so reversing the order of single and composite figures should eliminate the effect. On the other hand, the feature hijacking explanation suggests that first figures may be selectively reinforced or weakened by the presentation of the second. Accordingly, for instance, congruency between the first and second figures could 
strengthen the first as well as the second. In this case, reversing their order would not make a great difference.

Finally, reversing the first and second figures will allow us to evaluate the congruency effect of the single figure more closely. In Experiment 1, this figure showed a congruency effect in both presentation conditions, although it was only briefly presented. This effect may be explained by primacy; the congruency effect may be larger for the single figure because it was the first figure the participant saw. If the primacy explanation holds, then the composite figures should elicit larger congruency effects in the following experiment.

\section{EXPERIMENT 2}

\section{Method}

Participants. Fifteen participants ( 6 male) received a small payment for taking part; all had normal or corrected-to-normal vision. The mean age was 21 years (range, 18-27 years).

Design and Procedure. Experiment 2 was identical to Experiment 1 , with the following exceptions in design and procedure. The order of the single and composite figures was reversed; a trial started with a composite figure, then a single figure was presented, and finally the test pair appeared on the screen. The composite figure was presented for 50 or $500 \mathrm{msec}$ and the single figure for $50 \mathrm{msec}$.

\section{Results}

Erroneous responses constituted $5.0 \%$ of all trials. None of the participants showed a speed-accuracy trade-off, so error rates were not analyzed any further. The selection of trials for the RT analysis and the cutoff values were identical to those in Experiment 1; the factors of the ANOVA were also as in Experiment 1. Congruency of the single figure facilitated the task in both short $[F(1,6777)=4.91$, $p=.02]$ and long $[F(1,6755)=21.20, p<.001]$ presentation conditions. In addition, a main effect of test type was obtained for both presentation durations $[F(2,6777)=$ $226.19, p<.001$, for short and $F(2,6755)=202.85, p<$ .001 , for long presentations of the composite figure]. No

Table 2A

Mean Reaction Times (RTs, in Milliseconds; With Standard Deviations) in the Short (50-msec) Presentation Condition of Experiment 2 to Global (G), Local (L), and Mosaic (M) Test Pairs for Congruent (C) and Incongruent (I) Single and Composite Figures

\begin{tabular}{ccccc}
\hline & & & \multicolumn{2}{c}{ RT } \\
\cline { 3 - 5 } Single & Composite & Test Type & $M$ & $S D$ \\
\hline C & C & G & 470 & 144 \\
& & L & 485 & 141 \\
& I & M & 544 & 226 \\
& & G & 472 & 148 \\
& & L & 493 & 163 \\
I & C & G & 543 & 188 \\
& & L & 479 & 141 \\
& & M & 492 & 162 \\
& I & G & 542 & 193 \\
& & L & 480 & 171 \\
& & M & 591 & 152 \\
& & & & 196 \\
\hline
\end{tabular}

other effects approached significance. The mean RTs are shown in Tables $2 \mathrm{~A}$ and $2 \mathrm{~B}$ for short and long presentations of the composite figure.

\section{Discussion}

The main effect of test type was similar to that in Experiment 1 ; responses were fastest for global test pairs and slowest for mosaic ones, an effect of stimulus complexity. Again as in Experiment 1, RTs decreased with congruency of the single figure. This effect, therefore, occurs irrespective of the order of the single and composite figures. RT was unaffected by congruency of the composite figures, irrespective of their presentation duration. Primacy, therefore, does not guarantee congruency effects. Rather, the results suggest that the congruency effect for composite figures is more volatile than that for single figures and not robust to intervening stimuli.

No interaction between the congruency effects of first and second figures was observed, now that the composite preceded the single figure. Subsequent exposure to a possible interpretation of a composite figure did not influence the interpretation of that figure afterward. This result is in accordance with our interpretation of Experiment 1, that such influences occur during completion rather than afterward. The absence of this interaction also implies that the composite figure did not prime the subsequent single figure. In Sekuler and Palmer (1992), a composite figure did prime a subsequent single figure, but their result was obtained in conditions in which the single figure was the target of the task. We thus may consider that targetspecific processes, such as response selection, could have been responsible for the result. Alternatively, the sensitivity of our method may be less than theirs.

The effects of congruency of the single figure on different types of test pairs were indistinguishable. The selective effects in Experiment 1, in which global and local but not mosaic pairs were facilitated, may therefore have been due to the intervening composite figure. This outcome is in accordance with the understanding that in Experiment 1, the long presentation of the composite figure helped erase the mosaic figure from memory, because the occlusion interpretations were completed.

An alternative explanation for Experiment 1 was based on the interaction between low-level features of the first and second stimuli, irrespective of completion. According to this explanation, the first and second stimuli should still have interacted when their order was reversed. The absence of this interaction in Experiment 2, therefore, is evidence against such an explanation. In addition, this account attributed the disappearance of priming effects for mosaic figures under long presentation of the composite figure to hijacking the features of the mosaic figure. For the reversed stimulus order, feature hijacking would be expected, if at all, for long presentations of the composite figure rather than for short ones. As can be observed in Tables $2 \mathrm{~A}$ and $2 \mathrm{~B}$, however, short presentation times were rather where the congruency effect for mosaic figures was strongest. Thus, in both crucial cases, the results are oppo- 
Table 2B

Mean Reaction Times (RTs, in Milliseconds; With Standard Deviations) in the Long (500-msec) Presentation Condition of Experiment 2 to Global (G), Local (L), and Mosaic (M) Test Pairs for Congruent (C) and Incongruent (I) Single and Composite Figures

\begin{tabular}{ccccc}
\hline & & & \multicolumn{2}{c}{ RT } \\
\cline { 3 - 5 } Single & Composite & Test Type & $M$ & SD \\
\hline C & C & G & 469 & 173 \\
& & L & 487 & 133 \\
& & M & 531 & 196 \\
& I & G & 475 & 170 \\
& & L & 482 & 155 \\
I & C & G & 527 & 171 \\
& & L & 475 & 141 \\
& & M & 488 & 137 \\
& I & G & 548 & 196 \\
& & L & 473 & 135 \\
& & M & 547 & 142 \\
& & & 547 \\
\hline
\end{tabular}

site those that would be predicted on the basis of priming of occlusion-irrelevant low-level features.

It may still be argued, however, that in Experiment 2 an interaction between composite and single figures failed to occur because the single figure was presented too briefly to disambiguate the preceding composite figure. For instance, their interaction could be based on high-level semantic attributes of the figure that require a longer presentation duration to develop. In Experiment 3, we therefore varied the presentation time of the single figure while the order of the two figures remained the same as it was in Experiment 2.

\section{EXPERIMENT 3}

\section{Method}

Participants. Eleven participants received a small payment in return for taking part in the experiment. Eight of them were female, 3 male; all had normal or corrected-to-normal vision. Their ages ranged from 19 to 35 , around a mean of 22 years.

Design and Procedure. Experiment 3 was identical to Experiment 2, except for the presentation durations. The duration of the composite figure was fixed at $50 \mathrm{msec}$, and that of the single figure could be either 50 or $500 \mathrm{msec}$.

\section{Results}

Errors occurred in $3.4 \%$ of trials overall. No trade-off between RT and proportion correct was observed, and therefore only the RT data were analyzed further. The selection of trials for analysis and the cutoff values were identical to those in the previous experiments, and so was the design of the ANOVA. For long presentation of the single figure, there were main effects of single figure congruency $[F(1,4593)=53.95, p<.001]$ and type of test pair $[F(2,4593)=105.98, p<.001]$.

For short presentations also, congruency of the single figure resulted in faster responses $[F(1,4559)=19.16$, $p<.001]$. The main effect of test type $[F(2,4559)=$ 93.70, $p<.001]$ was qualitatively similar to that found in the other experiments, but it interacted with the effect of single figure $[F(2,4559)=3.21, p<.05]$. The congruency effect of the single figure was present for local and global test pairs, but not for mosaic ones (see Figure 6). There was no main effect of the composite figure, but its congruency had different effects for different test types

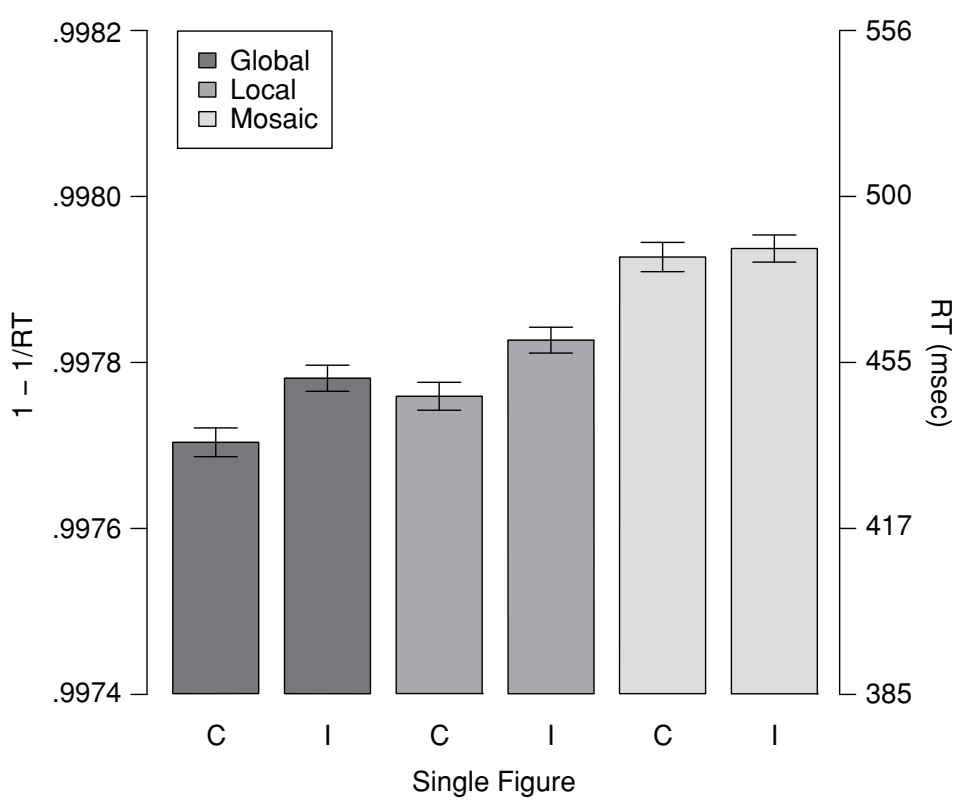

Figure 6. The interaction between test type (local, global, or mosaic) and congruency (congruent $[\mathrm{C}]$ or incongruent $[\mathrm{I}]$ ) of the single figure in the short (50msec) presentation condition of Experiment 3. Depicted are means and $95 \%$ confidence intervals of reaction times (RTs). 


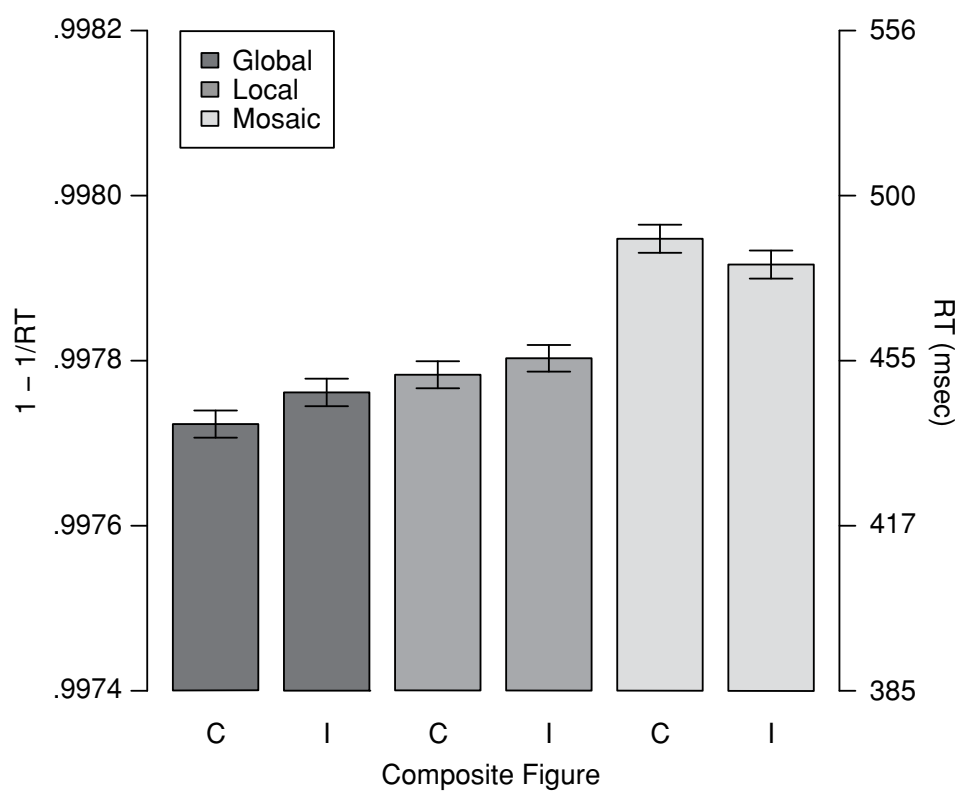

Figure 7. The interaction between test type (local, global, or mosaic) and congruency (congruent $[\mathrm{C}]$ or incongruent $[\mathrm{I}]$ ) of the composite figure in the short (50-msec) presentation condition of Experiment 3. Depicted are means and $\mathbf{9 5 \%}$ confidence intervals of reaction times (RTs).

$[F(2,4559)=3.12, p<.05]$. In this interaction, congruent composite figures facilitated responses to congruent global and local test pairs but slightly hindered those to mosaic pairs (see Figure 7). Mean RTs for the short and long presentation conditions are listed in Tables $3 \mathrm{~A}$ and $3 \mathrm{~B}$.

\section{Discussion}

The main effect of test type was similar to that in the previous two experiments, and so was the congruency effect of single figures. As in Experiment 2, there was no main effect of congruency for composite figures. This reiterates that the effects of these figures are weaker than those of the single figures, irrespective of presentation duration. Single figures are more easily perceived and better retained, facilitating subsequent responses more than composite figures.

In Experiment 3, we found that under long presentation conditions the single figure did not interact with the preceding composite figure. For long presentations, priming was equally effective for global, local, and mosaic figures, and congruency of the preceding composite figure did not modify this result. As in Experiment 2, single figures did not influence the perception of the composite figure afterward, even if they were presented for a long time. Neither did the briefly presented composite prime the subsequent single figures. In particular, its failure to selectively prime a mosaic single figure contrasts with the results of Sekuler and Palmer (1992).

When the single figures were briefly presented, two interaction effects were observed. In the first one, the congruency effect of the single figure was largest on global figures and absent for mosaic ones (see Figure 6). We may assume that for reasons similar to those in Experiment 1, if a mosaic is irrelevant to the composite figure because the completion has already been made, the mosaic figure is not available for priming even if it immediately precedes the task figures.

The second interaction showed that the congruency of composite figures was facilitatory for global, neutral for local, and inhibitory for mosaic test figures (see Figure 7). This result suggests a priming effect of the composite on the test figures. The facilitation of RTs with the global test figures suggests that a global completion of the first, composite figure primed those figures. The negative congruency effect for mosaic figures suggests that they became

Table 3A

Mean Reaction Times (RTs, in Milliseconds; With Standard Deviations) in the Short (50-msec) Presentation Condition of Experiment 3 to Global (G), Local (L), and Mosaic (M) Test Pairs for Congruent (C) and Incongruent (I) Single and Composite Figures

\begin{tabular}{ccccc}
\hline & & & \multicolumn{2}{c}{ RT } \\
\cline { 4 - 5 } Single & Composite & Test Type & $M$ & $S D$ \\
\hline C & C & G & 461 & 167 \\
& & L & 475 & 143 \\
& I & M & 518 & 164 \\
& & G & 470 & 188 \\
& L & 470 & 140 \\
I & M & 517 & 159 \\
& & G & 469 & 130 \\
& & L & 475 & 107 \\
& I & M & 524 & 158 \\
& & G & 480 & 135 \\
& & L & 494 & 161 \\
& & M & 508 & 169 \\
\hline
\end{tabular}


Table 3B

Mean Reaction Times (RTs, in Milliseconds; With Standard Deviations) in the Long (500-msec) Presentation Condition of Experiment 3 to Global (G), Local (L), and Mosaic (M) Test Pairs for Congruent (C) and Incongruent (I) Single and Composite Figures

\begin{tabular}{cccrr}
\hline & & & \multicolumn{2}{c}{ RT } \\
\cline { 3 - 5 } Single & Composite & Test Type & $M$ & $S D$ \\
\hline C & C & G & 441 & 97 \\
& & L & 454 & 99 \\
& I & M & 498 & 160 \\
& & G & 449 & 119 \\
& & L & 460 & 105 \\
I & C & G & 490 & 131 \\
& & G & 470 & 164 \\
& & I & 472 & 115 \\
& & G & 520 & 153 \\
& & L & 462 & 111 \\
& & M & 471 & 106 \\
& & & 506 & 133 \\
\hline
\end{tabular}

unpreferred once the completion of any preceding composite figure was made (see van Leeuwen \& Lachmann, 2004, for a detailed account of negative congruency effects).

The absence of these interactions under long presentation conditions for the single figure indicates that they are attenuated by intervening stimuli that are presented long enough. This is consistent with the restriction of the pivotal interaction in Experiment 1 to the short presentation of the second figure.

We note that these two interactions were not observed under the short presentation conditions in Experiment 2. These and the shorter Experiment 3 conditions were exactly the same; both composite and single figures were presented for $50 \mathrm{msec}$. However, in Experiment 2, the standard deviations of the mean RTs were higher on average than those in Experiment 3. Thus, the conditions of Experiment 2 may have not made these interactions detectable. One reason may be that the unpredictability in presentation time affected trials in Experiment 2 from the first stimulus, whereas in the present experiment this unpredictability arose only with the second stimulus.

We may consider the result for the short presentations a partial confirmation of Sekuler and Palmer (1992), who found that composite figures primed subsequent task figures. These authors found that briefly presented composites facilitated, rather than inhibited, responses to mosaics in the test pair. This difference can be ascribed to the long ISI and the absence of a mask in our study, which allowed completion to finish. As a result, brief presentations facilitated global figures, just as the long presentations in Sekuler and Palmer did.

\section{GENERAL DISCUSSION}

In three experiments, we investigated temporal context effects on the processing of composite figures. The experimental paradigm was a same-different task for shapes in which two primes, a single and a composite one, preceded a test pair. The composite figures could be understood as mosaics or as partially occluded shapes. Alternative local and global completions of these occluded shapes were possible. The single figures could be the unoccluded versions of these local and global completions (which were, by association, called local and global figures) or mosaic figures. The test pair was, again, a pair of global, local, or mosaic single figures.

A consistent effect found in all experiments was that RTs were fastest for global same-different pairs and slowest for mosaics, with RTs for local ones lying in between. The primary focus was on the changes in RTs as a function of the congruency of the two preceding figures, and specifically on the interaction of the two preceding figures.

We expected the preceding figures to prime the samedifferent task, based on congruency relations between the preceding and the test figures. For single figures, congruency almost always resulted in priming, but for composite figures this was predominantly the case when they were presented immediately prior to the test pair. This result suggests that the representation of the composite figures is more volatile than that of single figures. This theory is in line with the amodal character of their representation, since no visual sensation corresponded to the missing part. If the composite figure was presented immediately prior to the task figures, it could not prime them independently under brief presentation conditions. In such a case, priming could be achieved only if the first stimulus was congruent to the task figures also. We understood this pivotal effect in terms of resolved ambiguity; congruent preceding figures bias a particular interpretation (completion or mosaic) of the composite figure, and this allows it to exert a priming influence on the subsequent test pair.

A typical effect in the primed matching paradigm is that composite figures prime single ones in the task (Sekuler \& Palmer, 1992; van Lier, Leeuwenberg, \& van der Helm, 1995). In these experiments, the target of priming is also that of the task. We were able to replicate this effect, to some extent, in our Experiment 3. The difficulty with interpreting these results is that they could be ascribed to task-related decision processes. Our emphasis has been on detecting priming between nontargets. Our method, however, may have the drawback that it is less sensitive, and thereby misses important priming effects.

The possible lack of sensitivity of our task with respect to priming makes our central result even more remarkable. The combined effect of congruency of the first and second stimuli to the task figures is sometimes greater than the sum of its parts. This was the case when the first and second were both congruent to the task figures, and therefore congruent to each other as well. Their superadditive effect can be interpreted as priming between nontargets.

In our understanding, the effect of a single figure priming a congruent nontarget composite implies that the first biases the interpretation of the second, thereby removing its inherent ambiguity. We obtained this result for preceding local, global, and mosaic single figures. This means that temporal context could equally strengthen alternative 
occlusion interpretations, as well as mosaic representations of the composite figure. This result is in line with the effect of spatial context, in which surrounding shapes bias the interpretation of an occluded image (Rauschenberger et al., 2004), and it gives equal status to priming and occlusion interpretations (Bruno et al., 1997) during the process of completing the occluded stimulus.

On the other hand, after completion has finished, mosaic primes seem to lose their effectiveness more rapidly than local or global occlusion primes. When the composite is presented for a long duration, preceding local and global single figures still can have a priming influence on the task, but mosaic ones cannot. We considered this a result of the mosaic representation's being erased from memory after the completion interpretation of the composite stimulus had finished. This effect relates to preemption in visual search. In Experiment 3, for mosaic test figures a negative congruency effect of preceding composite figures was observed. This result, too, suggests that mosaics are not preferred once completion has finished (a partial replication of Sekuler \& Palmer, 1992). Although Sekuler and Palmer suggested a mosaic stage in perception, our present explanation differs: Mosaic and occlusion interpretations appear to have equal status insofar as both can be strengthened by priming during the processing of a compound stimulus. Which one will be chosen appears to be highly context-sensitive. However, once an occlusion interpretation is chosen, the representation of the mosaic part is disposed of, whereas alternative occlusion interpretations persist (van Lier, Leeuwenberg, \& van der Helm, 1995).

Our results show an asymmetry in time of temporal context effects on completion between nontarget figures. At least one study previously failed to find such effects (Pratt \& Sekuler, 2001). In their study, participants saw disjoint fragments that were subsequently occluded. After occlusion, the fragments could, and indeed were, represented as one whole object. This suggested that effects of prior exposure did not influence completion, but this result may have occurred because the occlusion lasted long enough (400 msec) to leave room for fragmented as well as completion interpretations to arise. In our study, effects of temporal context were clearly in evidence: Processing of composite figures may be influenced directly by a preceding single figure. When, however, the composite is shown before the single figure, the composite fails to have a similar influence, nor does the single influence the preceding composite figure. This is in line with the notion that completion is a quick and mandatory process (Rensink \& Enns, 1998) and confirms that a specific interpretation has been cemented after completion.

The flexibility of perception, therefore, comes not from representing composite figures as a collection of unrelated features that can be regrouped at will, but from the fact that several alternative completions may be temporarily available in parallel (Bruno et al., 1997; van Lier, van der Helm, \& Leeuwenberg, 1995). If such flexibility exists, we might expect that alternative representations of composites can prime alternative single figures, if the task requires this. It has indeed been demonstrated that ambiguous occlusions prime alternative single figures when they appear as task figures (van Lier, Leeuwenberg, \& van der Helm, 1995).

The notion that context influences the amodal completion of occluded figures is consistent with the pervasiveness of context effects on visual perception (Hochberg, 2003; Leeuwenberg, Mens, \& Calis, 1985). It has been demonstrated that image features are differentially processed on the neural level with changing context (Albright \& Stoner, 2002), and cells as early as V1 reflect past experience (Lee, Yang, Romero, \& Mumford, 2002). For composite figures, their interpretation may change depending on experimental context (Peterson \& Hochberg, 1983; Stins \& van Leeuwen, 1993), spatial context (Rauschenberger et al., 2004), and temporal context (Joseph \& Nakayama, 1999; Zemel et al., 2002). In addition, temporal context can directly influence ongoing completion processes.

\section{REFERENCES}

Albright, T. D., \& Stoner, G. R. (2002). Contextual influences on visual processing. Annual Review of Neuroscience, 25, 339-379.

Altmann, C. F., Bülthoff, H. H., \& Kourtzi, Z. (2003). Perceptual organization of local elements into global shapes in the human visual cortex. Current Biology, 13, 342-349.

Beller, H. K. (1971). Priming: Effects of advance information on matching. Journal of Experimental Psychology, 87, 176-182.

Bruno, N., Bertamini, M., \& Domini, F. (1997). Amodal completion of partly occluded surfaces: Is there a mosaic stage? Journal of Experimental Psychology: Human Perception \& Performance, 23, 1412-1426.

Buffart, H., \& Leeuwenberg, E. (1981). Coding theory of visual pattern completion. Journal of Experimental Psychology: Human Perception \& Performance, 7, 241-274.

Buffart, H., Leeuwenberg, E., \& Restle, F. (1983). Analysis of ambiguity in visual pattern completion. Journal of Experimental Psychology: Human Perception \& Performance, 9, 980-1000.

Gerbino, W., \& Salmaso, D. (1987). The effect of a modal completion on visual matching. Acta Psychologica, 65, 25-46.

Hoaglin, D. C., Mosteller, F., \& Tukey, J. W. (Eds.) (2000). Understanding robust and exploratory data analysis. New York: Wiley. (Original work published 1983)

Hochberg, J. (2003). Acts of perceptual inquiry: Problems for any stimulus-based simplicity theory. Acta Psychologica, 114, 215-228.

JosePH, J. S., \& NAKAYAMA, K. (1999). Amodal representation depends on the object seen before partial occlusion. Vision Research, 39, 283-292.

Kamitani, Y., \& Shimojo, S. (2004). Global yet early processing of visual surfaces. In L. M. Chalupa \& J. S. Werner (Eds.), The visual neurosciences. Cambridge, MA: MIT Press.

Kanizsa, G. (1979). Organization in vision: Essays on gestalt perception. New York: Praeger.

Kanizsa, G., \& Gerbino, W. (1982). Amodal completion: Seeing or thinking? In J. Beck (Ed.), Organization and representation in perception. Hillsdale, NJ: Erlbaum.

Kellman, P. J., \& Shipley, T. F. (1991). A theory of visual interpolation in object perception. Cognitive Psychology, 23, 141-221.

Lee, T. S., Yang, C. F., Romero, R. D., \& Mumford, D. (2002). Neural activity in early visual cortex reflects behavioral experience and higher-order perceptual saliency. Nature Neuroscience, 5, 589-597.

Leeuwenberg, E., Mens, L., \& Calis, G. (1985). Knowledge within perception: Masking caused by incompatible interpretation. Acta Psychologica, 59, 91-102.

Michotte, A., Thinès, G., \& Crabbé, G. (1964). Les compléments 
amodaux des structures perceptives (Studia Psychologica). Louvain: Publications universitaires de Louvain.

Nikolaev, A. R., \& VAn Leeuwen, C. (2004). Flexibility in spatial and non-spatial feature grouping: An event-related potentials study. Cognitive Brain Research, 22, 13-25.

Peterson, M. A., \& Hochberg, J. (1983). Opposed-set measurement procedure: A quantitative analysis of the role of local cues and intention in form perception. Journal of Experimental Psychology: Human Perception \& Performance, 9, 183-193.

Pinheiro, J. C., \& Bates, D. M. (2000). Mixed-effects models in S and $S$-PLUS. New York: Springer.

Plomp, G., Nakatani, C., Bonnardel, V., \& van Leeuwen, C. (2004). Amodal completion as reflected by gaze durations. Perception, 33, 1185-1200.

Pratt, J., \& Sekuler, A. B. (2001). The effects of occlusion and past experience on the allocation of object-based attention. Psychonomic Bulletin \& Review, 8, 721-727.

Rauschenberger, R., Peterson, M. A., Mosca, F., \& Bruno, N. (2004). Amodal completion in visual search: Preemption or contex effects? Psychological Science, 15, 351-355.

RAUSCHENBERGER, R., \& YANTIS, S. (2001). Masking unveils pre-amodal completion representation in visual search. Nature, 410, 369-372.

RENSINK, R. A., \& ENNS, J. T. (1998). Early completion of occluded objects. Vision Research, 38, 2489-2505.

Sekuler, A. B., \& Palmer, S. E. (1992). Perception of partly occluded objects: A microgenetic analysis. Journal of Experimental Psychology: General, 21, 95-111.

Sekuler, A. B., Palmer, S. E., \& Flynn, C. (1994). Local and global processes in visual completion. Psychological Science, 5, 260-267.

Shipley, T. F., \& Kellman, P. J. (2003). Boundary completion in il- lusory contours: Interpolation or extrapolation? Perception, 32, 985999

Stins, J. F., \& van Leeuwen, C. (1993). Context influence on the perception of figures as conditional upon perceptual organization strategies. Perception \& Psychophysics, 53, 34-42.

van Leeuwen, C., \& Lachmann, T. (2004). Negative and positive congruence effects in letters and shapes. Perception \& Psychophysics, 66, 908-925.

VAN Lier, R. J., Leeuwenberg, E. L. J., \& VAN der Helm, P. A. (1995). Multiple completions primed by occlusion patterns. Perception, 24, 727-740.

VAN Lier, R. J., VAN Der Helm, P. A., \& LeEuwenberG, E. L. J. (1995). Competing global and local completions in visual occlusion. Journal of Experimental Psychology: Human Perception \& Performance, 21, 571-583.

Zemel, R. S., Behrmann, M., \& Mozer, M. C. (2002). Experiencedependent perceptual grouping and object-based attention. Journal of Experimental Psychology: Human Perception \& Performance, 28, 202-217.

\section{NOTE}

1. A separate analysis on the effect of stimulus classes showed no differential priming effects for familiar and unfamiliar completions. There were main effects of familiarity of the test pair figures on RTs, but familiarity did not interact with the congruency of the simple or the composite figure.

(Manuscript received December 10, 2004; revision accepted for publication September 13, 2005.) 